
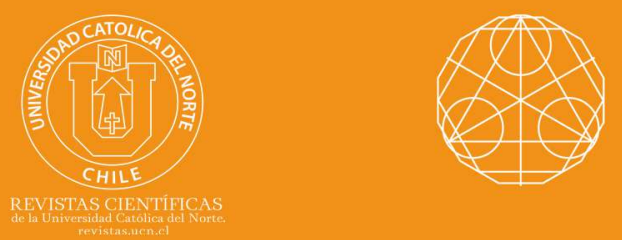

\title{
Creating a new two-step recursive memory method with eight-order based on Kung and Traub's method
}

Vali Torkashvand ${ }^{1}$ (응 orcid.org/0000-0001-8033-8279

Mohammad Momenzadeh ${ }^{2}$ (1) orcid.org/0000-0002-7830-9820

Taher Lotf ${ }^{3}$

${ }^{1}$ Islamic Azad University, Shahr-e-Qods Branch, Young Researchers and Elite Club, Tehran, Iran.

torkashvand1978@gmail.com

Islamic Azad University, Dept. of Applied Mathematics, Hamedan Branch, Hamedan, Iran.

2®momenzadeh12@yahoo.com; ${ }^{3}$ lotfi@iauh.acir

\section{Abstract:}

We are devoted to the study of an iterative recursive TraubSteffensen like method for approximating the simple roots of a nonlinear equation. Using the recursive technique, the R-order of convergence is increased from 4 to 8 without any new function evaluations, which means $100 \%$ improvement of the order of the convergence. The theoretical study of the convergence rate is investigated and demonstrated. A few nonlinear problems are presented to justify the theoretical study.

Keywords: Nonlinear equations; Simple roots; Computational order of convergence; Weight function; Recursive method with memory.

MSC (2020): 65G99, 49M15, 65H05.

\section{Cite this article as (IEEE citation style):}

V. Torkashvand, M. Momenzadeh, and T.Lotf, "Creating a new two-step recursive memory method with eight-order based on Kung and Traub's method", Proyecciones (Antofagasta, On line), vol. 39, no. 5, pp. 1167-1189, Oct. 2020, doi: 10.22199/issn.0717-6279-2020-05-0072.

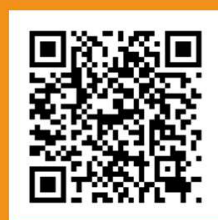

Article copyright: (C) 2020 Vali Torkashvand, Mohammad Momenzadeh, and Taher Lotf. This is an open access article distributed under the terms of the Creative Commons Licence, which permits unrestricted use and distribution provided the original author and source are credited.

(cc) BY 


\section{Introduction}

Solutions of nonlinear equations by iterative methods have been of great interest to numerical analysts. Iterative methods for approximating solutions of nonlinear equations are the most frequently used techniques. One can distinguish between two general approaches for solving nonlinear equations numerically, namely, one-point and multi-point methods. One of the popular methods for a single nonlinear equation $f(x)=0$, where $\xi$ is a single root, is written as:

$$
y_{k}=x_{k}-\frac{f\left(x_{k}\right)}{f^{\prime}\left(x_{k}\right)}, k=0,1,2, \cdots,
$$

which converges quadratically in some neighborhood of $\xi$. It is an optimal 1-point method with efficiency index of 1.41421. A great attention was paid to multi-point iterative methods; since they overcome on the theoretical limits of one-point methods concerning the order and efficiency index. In the following, we will first review a two-point iterative method. Ostrowski presented his two steps as follows:[17]

$$
\left\{\begin{array}{l}
y_{k}=x_{k}-\frac{f\left(x_{k}\right)}{f^{\prime}\left(x_{k}\right)}, k=0,1,2, \cdots, \\
x_{k+1}=y_{k}-\frac{f\left(x_{k}\right)}{f^{\prime}\left(x_{k}\right)} \frac{f\left(y_{k}\right)}{f\left(x_{k}\right)-2 f\left(y_{k}\right)} .
\end{array}\right.
$$

Then, Jarratt studied two-step without memory methods, an example of which is presented below [10]:

$$
\left\{\begin{array}{l}
w_{k}=\frac{f\left(x_{k}\right)}{f^{\prime}\left(x_{k}\right)}, y_{k}=x_{k}-\frac{1}{2} \frac{f\left(x_{k}\right)}{f^{\prime}\left(x_{k}\right)}, k=0,1,2, \cdots, \\
x_{k+1}=y_{k}+\frac{f\left(x_{k}\right)}{f^{\prime}\left(x_{k}\right)-3 f^{\prime}\left(x_{k}-\frac{2}{3} w_{k}\right)} .
\end{array}\right.
$$

A generalization of Ostrowski's method (1.3) was proposed by King in the form $[12]$

$$
\left\{\begin{array}{l}
y_{k}=x_{k}-\frac{f\left(x_{k}\right)}{f^{\prime}\left(x_{k}\right)}, k=0,1,2, \cdots, \\
x_{k+1}=y_{k}-\frac{f\left(y_{k}\right)}{f^{\prime}\left(x_{k}\right)} \frac{f\left(x_{k}\right)+\alpha f\left(y_{k}\right)}{f\left(x_{k}\right)+(\alpha-2) f\left(y_{k}\right)},
\end{array}\right.
$$

where $\alpha \in \mathbf{R}$ is a parameter. King's family (1.4) of two-point methods is optimal and has the order of convergence four. Likewise, Kung and Traub [14] have shown that the derivative two-point method has order four.

$$
\left\{\begin{array}{l}
y_{k}=x_{k}-\frac{f\left(x_{k}\right)}{f^{\prime}\left(x_{k}\right)}, k=0,1,2, \cdots, \\
x_{k+1}=y_{k}-\frac{f\left(x_{k}\right) f\left(y_{k}\right)}{\left(f\left(x_{k}\right)-f\left(y_{k}\right)\right)^{2}} \frac{f\left(x_{k}\right)}{f^{\prime}\left(x_{k}\right)},
\end{array}\right.
$$


The two-step family of Maheshwari, which was given in [16], is one of the most significant two-point optimal fourth-order methods:

$$
\left\{\begin{array}{l}
y_{k}=x_{k}-\frac{f\left(x_{k}\right)}{f^{\prime}\left(x_{k}\right)}, k=0,1,2, \cdots \\
x_{k+1}=x_{k}+\frac{1}{f^{\prime}\left(x_{k}\right)}\left(\frac{f\left(x_{k}\right)^{2}}{f\left(y_{k}\right)-f\left(x_{k}\right)}-\frac{f\left(y_{k}\right)^{2}}{f\left(x_{k}\right)}\right) .
\end{array}\right.
$$

Soleymani and Hosseinabadi [24] presented the following method for solving nonlinear equations:

$$
\left\{\begin{array}{l}
y_{k}=x_{k}-\frac{f\left(x_{k}\right)}{f\left[x_{k}, w_{k}\right]}, w_{k}=x_{k}-f\left(x_{k}\right), k=0,1,2, \cdots, \\
x_{k+1}=y_{k}-\frac{f\left(y_{k}\right)}{2 f\left[y_{k}, x_{k}\right]-f\left[w_{k}, x_{k}\right]}\left(1+f\left[w_{k}, x_{k}\right] \frac{f\left(y_{k}\right)}{f\left(w_{k}\right)}\right) .
\end{array}\right.
$$

Petkovic et al.'s method is the well-known iterative method with memory for finding $\xi$ by using

$$
\left\{\begin{array}{l}
y_{k}=x_{k}-\frac{f\left(x_{k}\right)}{\phi_{1}\left(x_{k}, \beta_{k}\right)}, u_{k}=\frac{f\left(y_{k}\right)}{f\left(x_{k}\right)}, v_{k}=\frac{f\left(y_{k}\right)}{f\left(x_{k}-\beta f\left(x_{k}\right)\right)}, k=0,1,2, \cdots, \\
\phi_{1}\left(x_{k}, \beta_{k}\right)=\frac{\left.f\left(x_{k}\right)-\beta_{k} f\left(x_{k}\right)\right)}{\beta_{k} f\left(x_{k}\right)}, h(0,0)=h_{u}(0,0)=h_{v}(0,0)=1, \\
h\left(u_{k}, v_{k}\right)=\frac{1+u_{k}}{1-v_{k}}, x_{k+1}=y_{k}-h\left(u_{k}, v_{k}\right) \frac{f\left(y_{k}\right)}{\phi_{1}\left(x_{k}, \beta_{k}\right)},
\end{array}\right.
$$

that converges $2+\sqrt{5} \approx 4.236$ and even $2+\sqrt{6} \approx 4.449$ in special cases, see Theorems 3 and 4 , respectively [18]. We state the following derivative free family of two-point methods with memory proposed by Cordero et al. [6],

$\left\{\begin{array}{l}\gamma_{0} \text { is given, } w_{k}=x_{k}+\gamma_{k} f\left(x_{k}\right), \gamma_{k}=\frac{-1}{N_{3}^{\prime}\left(x_{k}\right)}, y_{k}=\frac{f\left(x_{k}\right)}{f\left[x_{k}, w_{k}\right]}, k=0,1,2, \cdots, \\ x_{k+1}=y_{k}-\frac{f\left(y_{k}\right)}{\frac{f\left(y_{k}\right)-b f\left(x_{k}\right)}{y_{k}-x_{k}}+\frac{f\left(y_{k}\right)-(1-b) f\left(x_{k}\right)}{y_{k}-x_{k}}}, b \in \mathbf{R} .\end{array}\right.$

Each method of the family needs three function evaluations per full step and has order six. In the following, we state the following derivative free family of two-point methods with memory proposed by Cordero et al. [7],

$$
\left\{\begin{array}{l}
\gamma_{0}, x_{0}, p_{0}, \text { are given, } w_{0}=x_{0}+\gamma_{0} f\left(x_{0}\right), \gamma_{k}=\frac{-1}{N_{3}^{\prime}\left(x_{k}\right)}, p_{k}=-\frac{N_{4}^{\prime \prime}\left(w_{k}\right)}{-2 N_{4}^{\prime}\left(w_{k}\right)}, \\
y_{k}=x_{k}-\frac{f\left(x_{k}\right)}{f\left[x_{k}, w_{k}\right]+p_{k} f\left(w_{k}\right)}, w_{k}=x_{k}+\gamma_{k} f\left(x_{k}\right), k=0,1,2, \cdots, \\
x_{k+1}=y_{k}-\frac{f\left(y_{k}\right)}{f\left[x_{k}, y_{k}\right]+f\left[y_{k}, w_{k}\right]-f\left[x_{k}, w_{k}\right]+A\left(y_{k}-x_{k}\right)\left(y_{k}-w_{k}\right)} .
\end{array}\right.
$$


The R-order of convergence of the iterative methods (1.9) with memory is at least 7. Kansal et al.'s method [11] with memory as follows:

$$
\left\{\begin{array}{l}
\gamma_{k}=\frac{-1}{N_{3}^{\prime}\left(x_{k}\right)}, p_{k}=-\frac{N_{4}^{\prime \prime}\left(w_{k}\right)}{-2 N_{4}^{\prime}\left(w_{k}\right)}, k=1,2,3, \cdots, \\
w_{k}=x_{k}+\gamma_{k} f\left(x_{k}\right), t_{k}=\frac{f\left(y_{k}\right)}{f\left(x_{k}\right)}, g\left(t_{k}\right)=1-\frac{1+a}{2} t_{k}, a \in \mathbf{R}, k=0,1,2, \cdots, \\
y_{k}=x_{k}-\frac{f\left(x_{k}\right)}{f\left[x_{k}, w_{k}\right]+p_{k} f\left(w_{k}\right)}, x_{k+1} \\
=y_{k}-g\left(t_{k}\right) \frac{f\left(x_{k}\right)}{f\left[y_{k}, w_{k}\right]+p_{k} f\left(w_{k}\right)}\left(-1 \frac{a+1}{a+\left(1-\frac{2(a+1) f\left(y_{k}\right)}{f\left(x_{k}\right)}\right)^{1 / 2}}\right),
\end{array}\right.
$$

which has the convergence order 7 . Several two-step methods are proposed in $[9,13,20,22,23,25]$.

In this work, we develop a new three parameters two-point eight-order Traub-Steffensen type family of iterative methods. In other words, we consider an optimal without memory method, which uses three functional evaluations per iterate and has convergence order four. Then, without any additional functional evaluations, it is attempted to increase the convergence order of the given method by the technique of with memory method. This enables us to increase the efficiency index as well as convergence order. Another advantage of this method is that it can compete the two-step without memory methods since it has convergence order eight and only three functional evaluations, while the other uses three functional evaluations with order of convergence four. We have used the derivative version of Kung-Traub's in this paper.

This paper is organised as follows: In Section 2 we deal with introducing a two-step without memory method with the imposition of three self-accelerator parameters. In the following, in Section 3, we discuss how it could be a method with memory, by using a three suitable accelerators (which have been rarely used in these methods so far). As a consequence, in Section 3, introducing the free adaptation of free parameters by the method of interpolation of Newton's polynomials, we introduce recursive methods. Finally, in Section 4, the method is tested on well known numerical examples given in the literature. Numerical examples are given to test the efficiency and performance of the method. Numerical tests show that the new method is comparable with the well known existing methods and in many cases gives better results. Our results can be considered 
as an improvement and refinement of the previously known results in the literature.

\subsection{Definitions}

Before constructing the methods, here we state the following definitions.

Definition 1.1: Let $f(x)$ be a real function with a simple root $\alpha$, and let $\left\{x_{n}\right\}_{n=0}^{\infty}$ be a sequence of real numbers that converges towards $\alpha$. The order of convergence $r$ is given by

$$
\lim _{n \rightarrow \infty} \frac{x_{n+1}-\alpha}{\left(x_{n}-\alpha\right)^{r}}=A \neq 0,
$$

$A$ is the asymptotic error constant where $A \in \mathbf{R}^{+}[17]$.

The efficiency index is a criterion for comparing iterative methods of solving nonlinear equations.

Definition 1.2: Let $m$ be the number of function evaluations of the new method. The efficiency of the new method is measured by the concept of efficiency index [17] and defined as

$$
E I=r^{\frac{1}{m}}
$$

where $r$ is the order of convergence of the method. [19]:

In this work we use the symbol $\sim$ according to the following convention

If $\frac{f}{g} \rightarrow C$, where $C$ is a nonzero constant, we shall write $f=O(g)$ or $f \sim C g$.

\section{The methods and analysis of convergence}

In the following, we propose a general optimal class of fourth-order twopoint methods. By using a similar idea to that presented by Chun in $[2,3,4]$, we consider the Kung-Traub scheme (1.5). It is clear that formula (1.5) requires three evaluations per iteration and has an efficiency index of $4^{1 / 3}=$ 
1.58740. The purpose of this paper is to establish new derivative-free methods with eight order convergence and efficiency 2 ; hence, to derive maximum efficiency index. We consider approximating the derivative of the first step and second step by divided difference method:

$$
\left\{\begin{array}{l}
w_{k}=x_{k}+\beta f\left(x_{k}\right), f^{\prime}\left(x_{k}\right) \approx f\left[w_{k}, x_{k}\right]=\frac{f\left(w_{k}\right)-f\left(x_{k}\right)}{w_{k}-x_{k}} \\
f^{\prime}\left(y_{k}\right) \approx f\left[y_{k}, w_{k}\right]=\frac{f\left(y_{k}-f\left(w_{k}\right)\right.}{y_{k}-w_{k}} .
\end{array}\right.
$$

Kung-Traub method can be rewritten as follows:

$$
\left\{\begin{array}{l}
w_{k}=x_{k}+\beta f\left(x_{k}\right), y_{k}=x_{k}-\frac{f\left(x_{k}\right)}{f\left[w_{k}, x_{k}\right]}, k=0,1,2, \cdots, \\
x_{k+1}=y_{k}-\frac{f\left(x_{k}\right) f\left(y_{k}\right)}{\left(f\left(x_{k}\right)-f\left(y_{k}\right)\right)^{2}} \frac{f\left(x_{k}\right)}{f\left[y_{k}, w_{k}\right]} .
\end{array}\right.
$$

The scheme (2.2) uses three function evaluations and therefore is not an optimal two-point method. The error equation is:

$$
e_{k+1}=\left(-\left(1+\beta c_{1}\right)^{2} c_{2}^{2}\right) e_{k}^{3}+O\left(e_{k}^{4}\right),
$$

where we define $e_{k}=x_{k}-\xi$, be the error in the $k_{-}$th iterate and take into account $f(\xi)=0, c_{k}=f^{(k)}(\xi) / k ! f^{\prime}(\xi)$. Now, with the introduction of a weight function in the second step under the conditions given below, the convergence order of the without memory method (1.5) can be converted from three to four and converted into a without memory method with convergence order 4 . Let

$$
t=\frac{f(y)}{f(x)}, g(t)=g(0)+g^{\prime}(0) t+g^{\prime \prime}(0) \frac{t^{2}}{2 !}+g^{\prime \prime \prime}(0) \frac{t^{3}}{3 !}+\cdots .
$$

Thus, our new two-step derivative free iterative method without memory is given as follows:

$$
\left\{\begin{array}{l}
w_{k}=x_{k}+\beta f\left(x_{k}\right), y_{k}=x_{k}-\frac{f\left(x_{k}\right)}{f\left[w_{k}, x_{k}\right]}, k=0,1,2, \cdots, \\
x_{k+1}=y_{k}-g\left(t_{k}\right) \frac{f\left(x_{k}\right) f\left(y_{k}\right)}{\left(f\left(x_{k}\right)-f\left(y_{k}\right)\right)^{2}} \frac{f\left(x_{k}\right)}{f\left[y_{k}, w_{k}\right]} .
\end{array}\right.
$$

This method can also be rewritten by entering its two accelerator parameters, and a partial change in a two-step optimization method.

$(2.6)\left\{\begin{array}{l}w_{k}=x_{k}+\beta f\left(x_{k}\right), y_{k}=x_{k}-\frac{f\left(x_{k}\right)}{f\left[w_{k}, x_{k}\right]+\gamma f\left(w_{k}\right)}, k=0,1,2, \cdots, \\ x_{k+1}=y_{k}-g\left(t_{k}\right) \frac{f\left(x_{k}\right)^{2}}{\left(f\left(x_{k}\right)-f\left(y_{k}\right)\right)^{2}} \frac{f\left(y_{k}\right)}{f\left[y_{k}, w_{k}\right]+\gamma f\left(w_{k}\right)+\lambda\left(y_{k}-x_{k}\right)\left(y_{k}-w_{k}\right)} .\end{array}\right.$ 
To find the suitable weight functions $g\left(t_{k}\right)$ in (2.3), providing order four, we will use the method of undetermined coefficients and Taylor's series about zero, since $t$ tend to zero when $x$ tends to $\xi$. Theorem (2.1) demonstrates its convergence analysis.

Theorem 2.1. Assume that the functions $g, f: D \subset \mathbf{R} \rightarrow \mathbf{R}$ are four times continuously differentiable and $f$ has a simple zero $\xi \in D$. If $g(0)=$ $1, g^{\prime}(0)=-1,\left|g^{\prime \prime}(0)\right|<\infty$ and the initial point $x_{0}$ is sufficiently close to $\xi$, then the sequence $\left\{x_{n}\right\}_{n=0}^{\infty}$ defined by (2.6) converges to $\xi$ and the order of convergence is four.

Proof: By using Taylor's expansion of $f(x)$ about $\xi$ and taking into account that $f(\xi)=0$, we obtain

$$
f\left(x_{k}\right)=f^{\prime}(\xi)\left(e_{k}+c_{2} e_{k}^{2}+c_{3} e_{k}^{3}+c_{4} e_{k}^{4}+O\left(e_{k}^{5}\right)\right) .
$$

Then, computing $e_{k, w}=w_{k}-\xi$, we attain $w_{k}=x_{k}+\beta f\left(x_{k}\right)$

$$
e_{k, w}=e_{k}+e_{k} \beta f^{\prime}(\xi)\left(1+e_{k}\left(c_{2}+e_{k}\left(c_{3}+e_{k} c_{4}\right)\right)\right)+O\left(e_{k}^{5}\right),
$$

and

$$
\left\{\begin{aligned}
f\left(w_{k}\right)= & f^{\prime}(\xi)\left(e_{k}+e_{k} \beta f^{\prime}(\xi)\left(1+e_{k}\left(c_{2}+e_{k}\left(c_{3}+e_{k} c_{4}\right)\right)\right)+c_{2}\left(e_{k}+e_{k} \beta f^{\prime}(\xi)\right.\right. \\
& \left.\left(1+e_{k}\left(c_{2}+e_{k}\left(c_{3}+e_{k} c_{4}\right)\right)\right)\right)^{2}+c_{3}\left(e_{k}+e_{k} \beta f^{\prime}(\xi)\left(1+e_{k}\left(c_{2}+e_{k}\right.\right.\right. \\
& \left.\left.\left.\left.\left(c_{3}+e_{k} c_{4}\right)\right)\right)\right)^{3}+c_{4}\left(e_{k}+e_{k} \beta f^{\prime}(\xi)\left(1+e_{k}\left(c_{2}+e_{k}\left(c_{3}+e_{k} c_{4}\right)\right)\right)\right)^{4}\right) .
\end{aligned}\right.
$$

Considering $f[x, y]=\frac{f(x)-f(y)}{x-y}$ is Newton's first order divided difference. we get

$$
\left\{\begin{aligned}
f\left[x_{k}, w_{k}\right]= & -1 /\left(e_{k} \beta f^{\prime}(\xi)\left(1+e_{k}\left(c_{2}+e_{k}\left(c_{3}+e_{k} c_{4}\right)\right)\right)\right)^{-1}\left(e _ { k } f ^ { \prime } ( \xi ) \left(1+e_{k}\left(c_{2}+e_{k}\left(c_{3}\right.\right.\right.\right. \\
& \left.\left.\left.+e_{k} c_{4}\right)\right)\right)-f^{\prime}(\xi)\left(e_{k}+e_{k} \beta f^{\prime}(\xi)\left(1+e_{k}\left(c_{2}+e_{k}\left(c_{3}+e_{k} c_{4}\right)\right)\right)+c_{2}\left(e_{k}+e_{k} \beta\right.\right. \\
& \left.f^{\prime}(\xi)\left(1+e_{k}\left(c_{2}+e_{k}\left(c_{3}+e_{k} c_{4}\right)\right)\right)\right)^{2}+c_{3}\left(e_{k}+e_{k} \beta f^{\prime}(\xi)\left(1+e_{k}\left(c_{2}+e_{k}\left(c_{3}\right.\right.\right.\right. \\
& \left.\left.\left.\left.\left.\left.+e_{k} c_{4}\right)\right)\right)\right)^{3}+c_{4}\left(e_{k}+e_{k} \beta f^{\prime}(\xi)\left(1+e_{k}\left(c_{2}+e_{k}\left(c_{3}+e_{k} c_{4}\right)\right)\right)\right)^{4}\right)\right) .
\end{aligned}\right.
$$

Using the second step of (2.3), we attain

$$
\left\{\begin{aligned}
y_{k}= & \xi+\left(1+\beta f^{\prime}(\xi)\right)\left(\gamma-c_{2}\right) e_{k}^{2}+\left(\left(2+\beta f^{\prime}(\xi)\left(2+\beta f^{\prime}(\xi)\right)\left(q-c_{2}\right) c_{2}+\left(1+\beta f^{\prime}(\xi)\right)\right.\right. \\
& \left(2+\beta f^{\prime}(\xi)\right) c_{3} e_{k}^{3}+\left(-\gamma\left(5+\beta f^{\prime}(\xi)\left(7+5 \beta f^{\prime}(\xi)\left(4+\beta f^{\prime}(\xi)\right)\right)\right) c_{2}^{2}+\left(4+\beta f^{\prime}(\xi)\right.\right. \\
& \left.\left.\left(3+\beta f^{\prime}(\xi)\right)\right)\right) c_{2}^{3}+\gamma\left(4+\beta f^{\prime}(\xi)\left(7+\beta f^{\prime}(\xi)\left(5+\beta f^{\prime}(\xi)\right)\right)\right) c_{3}-\left(7+\beta f^{\prime}(\xi)(10+\right. \\
& \left.\left.\beta f^{\prime}(\xi)\left(7+2 \beta f^{\prime}(\xi)\right)\right)\right) c_{2} c_{3}+\left(1+\beta f^{\prime}(\xi)\right)\left(3+\beta f^{\prime}(\xi)\left(3+\beta f^{\prime}(\xi)\right)\right) c_{4} e_{k}^{4}+O\left(e_{k}^{5}\right) .
\end{aligned}\right.
$$


By putting $g(0)=1, g^{\prime}(0)=-1$, the final error expression is given by:

$$
\begin{aligned}
& e_{k+1}=\left(1+\beta f^{\prime}(\xi)\right)^{2}\left(\gamma+c_{2}\right)\left(f^{\prime}(\xi) g^{\prime \prime}(0)\left(1+\beta f^{\prime}(\xi)\right) \gamma^{2}-2 \lambda+f^{\prime}(\xi) c_{2}\left(2 \left(-2+g^{\prime \prime}(0)\right.\right.\right. \\
& \left.\left.\left.\left.+g^{\prime \prime}(0) f^{\prime}(\xi) \beta\right) \gamma+\left(-4+g^{\prime \prime}(0)+g^{\prime \prime}(0) f^{\prime}(\xi) \beta\right) c_{2}\right)+2 f^{\prime}(\xi) c_{3}\right)\right)\left(2 f^{\prime}(\xi)\right)^{-1} e_{k}^{4}+O\left(e_{k}^{5}\right),
\end{aligned}
$$

which finishes the proof of the theorem.

Some other simple forms of functions $g$ can be:

$$
\left\{\begin{array}{l}
g_{1}(t)=1-t, g_{2}(t)=\frac{1}{1+t}, g_{3}(t)=\left(1-\frac{t}{2}\right)^{2}, g_{4}(t)=e^{-t} \\
g_{5}(t)=\frac{1+2 t}{1+3 t}, g_{6}(t)=\cos (t)-\sin (t), g_{7}(t)=\frac{1}{1+t+d t^{2}}, d \in \mathbf{R}
\end{array}\right.
$$

According to (2.5), we can produce any desire method of optimal order four by using only three functional evaluations per full cycle. Hence, we can have some contributed examples:

$\left(2.14\left\{\begin{array}{l}w_{k}=x_{k}+\beta f\left(x_{k}\right), y_{k}=x_{k}-\frac{f\left(x_{k}\right)}{f\left[w_{k}, x_{k}\right]+\gamma f\left(w_{k}\right)}, k=0,1,2, \cdots, \\ x_{k+1}=y_{k}-\left(1-\frac{f\left(y_{k}\right)}{f\left(x_{k}\right)}\right) \frac{f\left(x_{k}\right)^{2}}{\left(f\left(x_{k}\right)-f\left(y_{k}\right)\right)^{2}} \frac{f\left(y_{k}\right)}{f\left[y_{k}, w_{k}\right]+\gamma f\left(w_{k}\right)+\lambda\left(y_{k}-x_{k}\right)\left(y_{k}-w_{k}\right)},\end{array}\right.\right.$

with error equation:

$$
e_{k+1}=\frac{\left.\left(1+\beta f^{\prime}(\xi)\right)^{2}\left(\gamma+c_{2}\right)\left(\lambda+2 f^{\prime}(\xi) c_{2}\left(\gamma+c_{2}\right)-f^{\prime}(\xi) c_{3}\right)\right)}{f^{\prime}(\xi)} e_{k}^{4}+O\left(e_{k}^{5}\right),
$$

and, we find out new two-point methods without memory as follows:

$$
\left\{\begin{array}{l}
w_{k}=x_{k}+\beta f\left(x_{k}\right), y_{k}=x_{k}-\frac{f\left(x_{k}\right)}{f\left[w_{k}, x_{k}\right]+\gamma f\left(w_{k}\right)}, k=0,1,2, \cdots, \\
x_{k+1}=y_{k}-\left(\frac{1}{1+\frac{f\left(y_{k}\right)}{f\left(x_{k}\right)}}\right) \frac{f\left(x_{k}\right)^{2}}{\left(f\left(x_{k}\right)-f\left(y_{k}\right)\right)^{2}} \frac{f\left(y_{k}\right)}{f\left[y_{k}\right]+\gamma f\left(w_{k}\right)+\lambda\left(y_{k}-x_{k}\right)\left(y_{k}-w_{k}\right)},
\end{array}\right.
$$

and, its very simple error equation comes next as:

$$
\begin{gathered}
e_{k+1}=-\left(1+\beta f^{\prime}(\xi)\right)^{2}\left(\gamma+c_{2}\right)\left(f^{\prime}(\xi)\left(1+\beta f^{\prime}(\xi)\right) \gamma^{2}-\lambda+f^{\prime}(\xi)\left(2 f^{\prime}(\xi) \beta \gamma c_{2}+(-1\right.\right. \\
\left.\left.\left.\left.\left.f^{\prime}(\xi) \beta\right) \gamma\right) c_{2}^{2}\right)+c_{3}\right)\right)\left(f^{\prime}(\xi)\right)^{-1} e_{k}^{4}+O\left(e_{k}^{5}\right),
\end{gathered}
$$

or a new without memory method is given by :

$$
\left\{\begin{array}{l}
w_{k}=x_{k}+\beta f\left(x_{k}\right), y_{k}=x_{k}-\frac{f\left(x_{k}\right)}{f\left[w_{k}, x_{k}\right]+\gamma f\left(w_{k}\right)}, k=0,1,2, \cdots, \\
\left.x_{k+1}=y_{k}-\left(1-\frac{f\left(y_{k}\right)}{2 f\left(x_{k}\right)}\right)^{2}\right) \frac{f\left(x_{k}\right)^{2}}{\left(f\left(x_{k}\right)-f\left(y_{k}\right)\right)^{2}} \frac{f\left(y_{k}\right)}{f\left[y_{k}, w_{k}\right]+\gamma f\left(w_{k}\right)+\lambda\left(y_{k}-x_{k}\right)\left(y_{k}-w_{k}\right)},
\end{array}\right.
$$


with error equation:

$$
\begin{aligned}
e_{k+1} & =\left(1+\beta f^{\prime}(\xi)\right)^{2}\left(\gamma+c_{2}\right)\left(f^{\prime}(\xi)\left(1+\beta f^{\prime}(\xi)\right) \gamma^{2}-4 \lambda+f^{\prime}(\xi) c_{2}\left(2\left(-3+f^{\prime}(\xi) \beta\right)\right.\right. \\
& \left.\left.\left.\gamma+\left(7+f^{\prime}(\xi) \beta\right) c_{2}\right)+4 f^{\prime}(\xi) c_{3}\right)\right)\left(-f^{\prime}(\xi)\right)^{-1} e_{k}^{4}+O\left(e_{k}^{5}\right),
\end{aligned}
$$

or the following efficient method:

$$
\left\{\begin{array}{l}
w_{k}=x_{k}+\beta f\left(x_{k}\right), y_{k}=x_{k}-\frac{f\left(x_{k}\right)}{f\left[w_{k}, x_{k}\right]+\gamma f\left(w_{k}\right)}, k=0,1,2, \cdots, \\
x_{k+1}=y_{k}-e^{-\frac{f\left(y_{k}\right)}{f\left(x_{k}\right)}} \frac{f\left(x_{k}\right)^{2}}{\left(f\left(x_{k}\right)-f\left(y_{k}\right)\right)^{2}} \frac{f\left(y_{k}\right)}{f\left[y_{k}, w_{k}\right]+\gamma f\left(w_{k}\right)+\lambda\left(y_{k}-x_{k}\right)\left(y_{k}-w_{k}\right)} .
\end{array}\right.
$$

The error relation of (2.18) is of the form:

$$
\begin{aligned}
e_{k+1} & =\left(1+\beta f^{\prime}(\xi)\right)^{2}\left(\gamma+c_{2}\right)\left(f^{\prime}(\xi)\left(1+\beta f^{\prime}(\xi)\right) \gamma^{2}-2 \lambda+f^{\prime}(\xi) c_{2}\left(2\left(-1+f^{\prime}(\xi) \beta\right)\right.\right. \\
& \left.\left.\left.\gamma+\left(-3+f^{\prime}(\xi) \beta\right) c_{2}\right)+2 f^{\prime}(\xi) c_{3}\right)\right)\left(-2 f^{\prime}(\xi)\right)^{-1} e_{k}^{4}+O\left(e_{k}^{5}\right),
\end{aligned}
$$

is its error relation;or the new family of three-parametric methods is given by:

$$
\left\{\begin{array}{l}
w_{k}=x_{k}+\beta f\left(x_{k}\right), y_{k}=x_{k}-\frac{f\left(x_{k}\right)}{f\left[w_{k}, x_{k}\right]+\gamma f\left(w_{k}\right)}, k=0,1,2, \cdots \\
x_{k+1}=y_{k}-\frac{1+2 \frac{f\left(y_{k}\right)}{f\left(x_{k}\right)}}{1+3 \frac{f\left(y_{k}\right)}{f\left(x_{k}\right)}} \frac{f\left(x_{k}\right)^{2}}{\left(f\left(x_{k}\right)-f\left(y_{k}\right)\right)^{2}} \frac{f\left(y_{k}\right)}{f\left[y_{k}, w_{k}\right]+\gamma f\left(w_{k}\right)+\lambda\left(y_{k}-x_{k}\right)\left(y_{k}-w_{k}\right)},
\end{array}\right.
$$

with the follow-up error equation:

$$
\begin{aligned}
e_{k+1} & =\left(1+\beta f^{\prime}(\xi)\right)^{2}\left(\gamma+c_{2}\right)\left(3 f^{\prime}(\xi)\left(1+\beta f^{\prime}(\xi)\right) \gamma^{2}-\lambda+f^{\prime}(\xi)\left(c_{2}\left(4 \gamma+6 f^{\prime}(\xi) \beta\right)\right.\right. \\
& \left.\left.\left.\gamma+\left(-3+f^{\prime}(\xi) \beta\right)+c_{2}+3 f^{\prime}(\xi) c_{2} \beta\right)+c_{3}\right)\right)\left(-f^{\prime}(\xi)\right)^{-1} e_{k}^{4}+O\left(e_{k}^{5}\right),
\end{aligned}
$$

is its error relation;or derivative-free two-point method of fourth order:

$$
\begin{aligned}
& w_{k}=x_{k}+\beta f\left(x_{k}\right), y_{k}=x_{k}-\frac{f\left(x_{k}\right)}{f\left[w_{k}, x_{k}\right]+\gamma f\left(w_{k}\right)}, k=0,1,2, \cdots, \\
& x_{k+1}=y_{k}-\left(\cos \left(\frac{f\left(y_{k}\right)}{f\left(x_{k}\right)}\right)-\sin \left(\frac{f\left(y_{k}\right)}{f\left(x_{k}\right)}\right)\right) \frac{f\left(x_{k}\right)^{2}}{\left(f\left(x_{k}\right)-f\left(y_{k}\right)\right)^{2}} \frac{f\left(y_{k}\right)}{f\left[y_{k}, w_{k}\right]+\gamma f\left(w_{k}\right)+\lambda\left(y_{k}-x_{k}\right)\left(y_{k}-w_{k}\right)} .
\end{aligned}
$$

The error relation of $(2.20)$ is of the form:

$$
\begin{aligned}
e_{k+1} & =\left(1+\beta f^{\prime}(\xi)\right)^{2}\left(\gamma+c_{2}\right)\left(f^{\prime}(\xi)\left(1+\beta f^{\prime}(\xi)\right) \gamma^{2}+2 \lambda+f^{\prime}(\xi) c_{2}\left(2\left(3+f^{\prime}(\xi) \beta\right) \gamma\right.\right. \\
& \left.\left.\left.+\left(5+f^{\prime}(\xi) \beta\right) c_{2}\right)-2 f^{\prime}(\xi) c_{3}\right)\right)\left(2 f^{\prime}(\xi)\right)^{-1} e_{k}^{4}+O\left(e_{k}^{5}\right),
\end{aligned}
$$

and, we find out new two-point methods without memory as follows:

$$
\left\{\begin{array}{l}
w_{k}=x_{k}+\beta f\left(x_{k}\right), y_{k}=x_{k}-\frac{f\left(x_{k}\right)}{f\left[w_{k}, x_{k}\right]+\gamma f\left(w_{k}\right)}, k=0,1,2, \cdots, d \in \mathbf{R}, \\
x_{k+1}=y_{k}-\left(\frac{1}{1+\frac{f\left(y_{k}\right)}{f\left(x_{k}\right)}+d\left(\frac{f\left(y_{k}\right)}{f\left(x_{k}\right)}\right)^{2}}\right) \frac{f\left(x_{k}\right)^{2}}{\left(f\left(x_{k}\right)-f\left(y_{k}\right)\right)^{2}} \frac{f\left(y_{k}\right)}{f\left[y_{k}, w_{k}\right]+\gamma f\left(w_{k}\right)+\lambda\left(y_{k}-x_{k}\right)\left(y_{k}-w_{k}\right)},
\end{array}\right.
$$

and its very simple error equation comes next as: 


$$
\begin{aligned}
e_{k+1} & =\left(1+\beta f^{\prime}(\xi)\right)^{2}\left(\gamma+c_{2}\right)\left(( - 1 + d ) \left(f^{\prime}(\xi)\left(1+\beta f^{\prime}(\xi)\right) \gamma^{2}+\lambda+f^{\prime}(\xi) c_{2}(2(d+(-1\right.\right. \\
& \left.\left.\left.\left.+d) f^{\prime}(\xi) \beta\right) \gamma+\left(1+d+(-1+d) f^{\prime}(\xi) \beta\right) c_{2}\right)-f^{\prime}(\xi) c_{3}\right)\right)\left(f^{\prime}(\xi)\right)^{-1} e_{k}^{4}+O\left(e_{k}^{5}\right) .
\end{aligned}
$$

This error equation has three free parameters. Using the idea of forming with memory methods, one can approximate the parameters by using different methods, such as Secant [19, 21], Secant-like [27,28], Hermite interpolation [29] Newton's interpolation [15, 26]. In the remaining of this article, we present a new recursive technique to convert without memory method to with memory method. The efficiency index $(8)^{1 / 3}=2$, of the new method is higher than that of any existing two-step iterative methods. We update $\beta_{k}, \gamma_{k}$ and $\lambda_{k}$ based on all the available information from the first iteration to the current iteration. In the next section, the details will be given.

\section{Recursive method with memory}

Replacing the parameters $\beta, \gamma$ and $\lambda$ of methods (2.14), (2.16), (2.17), (2.18), (2.19), (2.20) and (2.21) with corresponding parameters $\beta_{k}, \gamma_{k}$ and $\lambda_{k}$, respectively, we can improve the convergence order of the methods (2.14), (2.16), (2.17), (2.18), (2.19), (2.20) and (2.21) and obtain the new iterative methods with memory. The parameters $\beta_{k}, \gamma_{k}$ and $\lambda_{k}$ can be calculated by Newton's interpolating polynomials, which depend on the data available in the current and the previous iterations. Using different selfaccelerating parameters, we can get different convergence orders of iterative methods with memory. In all of these cases, the parameters $\beta_{k}$ and $\gamma_{k}$ are the same and the difference in the calculation is $\lambda_{k}$. Therefore, each of the methods mentioned in relations $(2.14),(2.16),(2.17),(2.18),(2.19),(2.20)$ and (2.21) can be converted into a recursive manner in a two-point, three parameters self-accelerating, with memroy recursive method, respectively. To construct a recursive method with memory, we use the information not only in the current and its previous iterations, but also in all the previous iterations, i.e., from the beginning to the current iteration. The four methods $(2.14),(2.16),(2.19)$ and $(2.21)$ can be converted as follows into a with memory method, 


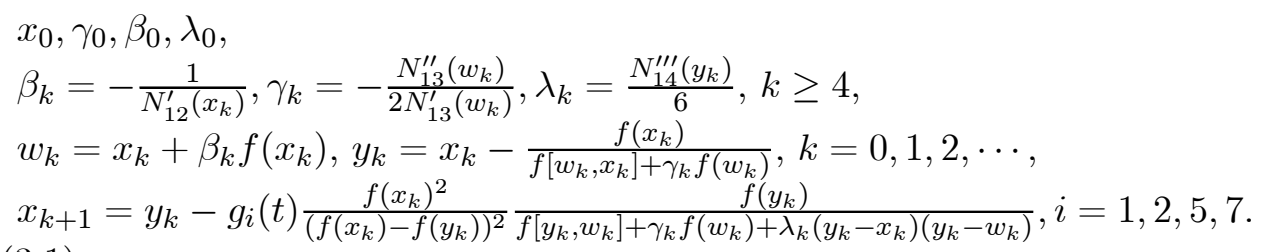

As an illustration, here we can define:

$\left\{\begin{array}{l}N_{3 k}(t)=N_{3 k}\left(t ; x_{k}, x_{k-1}, w_{k-1}, y_{k-1}, \ldots, x_{1}, w_{1}, y_{1}, x_{0}, w_{0}, y_{0}\right), k \in \mathbf{N}, \\ N_{3 k+1}(t)=N_{3 k+1}\left(t ; w_{k}, x_{k}, x_{k-1}, w_{k-1}, y_{k-1}, \ldots, x_{1}, w_{1}, y_{1}, x_{0}, w_{0}, y_{0}\right), \\ N_{3 k+2}(t)=N_{3 k+2}\left(t ; y_{k}, w_{k}, x_{k}, x_{k-1}, w_{k-1}, y_{k-1}, \ldots, x_{1}, w_{1}, y_{1}, x_{0}, w_{0}, y_{0}\right),\end{array}\right.$ $(3.2)$

where

$-N_{3}(t)=N_{3}\left(t ; x_{k}, x_{k-1}, w_{k-1}, y_{k-1}\right)$, as an interpolation polynomial of third degree, passing through the best four saved points $x_{k}, x_{k-1}, w_{k-1}, y_{k-1}$, for any $k \geq 1$.

$-N_{4}(t)=N_{4}\left(t ; w_{k}, x_{k}, x_{k-1}, w_{k-1}, y_{k-1}\right)$, as an interpolation polynomial of fourth degree, passing through the best fifth saved points $w_{k}, x_{k}, x_{k-1}, w_{k-1}, y_{k-1}$, for any $k \geq 1$.

$-N_{5}(t)=N_{5}\left(t ; y_{k}, w_{k}, x_{k}, x_{k-1}, w_{k-1}, y_{k-1}\right)$, as an interpolation polynomial of five degree, passing through the best six saved points $y_{k}, w_{k}, x_{k}, x_{k-1}, w_{k-1}, y_{k-1}$, for any $k \geq 1$.

The interpolation polynomials for other degrees can be defined in a similar way. Also methods $(2.17),(2.18)$ and $(2.20)$ can be converted to a recursive method as follows:

$$
\left\{\begin{array}{l}
x_{0}, \gamma_{0}, \beta_{0}, \lambda_{0}, \\
\beta_{k}=-\frac{1}{N_{12}^{\prime}\left(x_{k}\right)}, \gamma_{k}=-\frac{N_{13}^{\prime \prime}\left(w_{k}\right)}{2 N_{13}^{\prime}\left(w_{k}\right)}, \lambda_{k}=\frac{N_{14}^{\prime \prime}\left(y_{k}\right) N_{14}^{\prime \prime}\left(y_{k}\right)}{12}, k \geq 4, \\
w_{k}=x_{k}+\beta_{k} f\left(x_{k}\right), y_{k}=x_{k}-\frac{f\left(x_{k}\right)}{f\left[w_{k}, x_{k}\right]+\gamma_{k} f\left(w_{k}\right)}, k=0,1,2, \cdots, \\
x_{k+1}=y_{k}-g_{i}(t) \frac{f\left(x_{k}\right)^{2}}{\left(f\left(x_{k}\right)-f\left(y_{k}\right)\right)^{2}} \frac{f\left(y_{k}\right)}{f\left[y_{k}, w_{k}\right]+\gamma_{k} f\left(w_{k}\right)+\lambda_{k}\left(y_{k}-x_{k}\right)\left(y_{k}-w_{k}\right)}, i=3,4,6,
\end{array}\right.
$$

In the following, we discuss the general convergence analysis of the recursive methods with memory (3.1) and (3.3). It should be noted that the 
convergence order varies as the iteration go ahead. First, we introduce the following technical lemma.

Lemma 3.1. If $\beta_{k}=-\frac{1}{N_{3 k}^{\prime}\left(x_{k}\right)}, \gamma_{k}=-\frac{N_{3 k+1}^{\prime \prime}\left(w_{k}\right)}{2 N_{3 k+1}^{\prime}\left(w_{k}\right)}$ and $\lambda_{k}=\frac{N_{3 k+2}^{\prime \prime \prime}\left(y_{k}\right)}{6}$ or $\lambda_{k}=\frac{N_{3 k+2}^{\prime \prime \prime}\left(y_{k}\right) N_{3 k+2}^{\prime \prime}\left(y_{k}\right)}{12}$ with $N_{i}^{\prime}, i=3 k, 3 k+1,3 k+2$ defined as in (3.1) and (3.3) then the following equalities:

$$
\begin{aligned}
\left(1+\beta_{k} f^{\prime}(\xi)\right) & \sim \prod_{s=0}^{k-1} e_{s} e_{s, w} e_{s, y} \\
\left(c_{2}+\gamma_{k}\right) & \sim \prod_{s=0}^{k-1} e_{s} e_{s, w} e_{s, y}
\end{aligned}
$$

$$
\left.\left(\lambda_{k}+2 f^{\prime}(\xi) c_{2}\left(\gamma_{k}+c_{2}\right)-f^{\prime}(\xi) c_{3}\right)\right) \sim \prod_{s=0}^{k-1} e_{s} e_{s, w} e_{s, y}
$$

$\left((-1+d)\left(f^{\prime}(\xi)\left(1+\beta_{k} f^{\prime}(\xi)\right) \gamma_{k}^{2}+\lambda_{k}+f^{\prime}(\xi) c_{2}\left(2\left(d+(-1+d) f^{\prime}(\xi) \beta_{k}\right) \gamma_{k}\right.\right.\right.$ $\left.\left.\left.+\left(1+d+(-1+d) f^{\prime}(\xi) \beta_{k}\right) c_{2}\right)-f^{\prime}(\xi) c_{3}\right)\right) \sim \prod_{s=0}^{k-1} e_{s} e_{s, w} e_{s, y}$,

$$
\begin{aligned}
& \left(f^{\prime}(\xi)\left(1+\beta_{k} f^{\prime}(\xi)\right) \gamma_{k}^{2}-4 \lambda_{k}+f^{\prime}(\xi) c_{2}\left(2\left(-3+f^{\prime}(\xi) \beta\right) \gamma_{k}+\left(7+f^{\prime}(\xi) \beta_{k}\right) c_{2}\right)\right. \\
& \left.\left.+4 f^{\prime}(\xi) c_{3}\right)\right) \sim \prod_{s=0}^{k-1} e_{s} e_{s, w} e_{s, y}
\end{aligned}
$$

$\left(f^{\prime}(\xi)\left(1+\beta_{k} f^{\prime}(\xi)\right) \gamma_{k}^{2}-2 \lambda_{k}+f^{\prime}(\xi) c_{2}\left(2\left(-1+f^{\prime}(\xi) \beta_{k}\right) \gamma_{k}+\left(-3+f^{\prime}(\xi) \beta_{k}\right) c_{2}\right)\right.$ $\left.\left.+2 f^{\prime}(\xi) c_{3}\right)\right) \sim \prod_{s=0}^{k-1} e_{s} e_{s, w} e_{s, y}$,

$\left(3 f^{\prime}(\xi)\left(1+\beta_{k} f^{\prime}(\xi)\right) \gamma_{k}^{2}-\lambda_{k}+f^{\prime}(\xi)\left(c_{2}\left(4 \gamma_{k}+6 f^{\prime}(\xi) \beta_{k}\right) \gamma_{k}+\left(-3+f^{\prime}(\xi) \beta_{k}\right)\right.\right.$ $\left.\left.\left.+c_{2}+3 f^{\prime}(\xi) c_{2} \beta_{k}\right)+c_{3}\right)\right) \sim \prod_{s=0}^{k-1} e_{s} e_{s, w} e_{s, y}$,

$\left(f^{\prime}(\xi)\left(1+\beta_{k} f^{\prime}(\xi)\right) \gamma_{k}^{2}+2 \lambda_{k}+f^{\prime}(\xi) c_{2}\left(2\left(3+f^{\prime}(\xi) \beta_{k}\right) \gamma_{k}+\left(5+f^{\prime}(\xi) \beta_{k}\right) c_{2}\right)\right.$ $\left.\left.-2 f^{\prime}(\xi) c_{3}\right)\right) \sim \prod_{s=0}^{k-1} e_{s} e_{s, w} e_{s, y}$

where $e_{s}=x_{s}-\xi, e_{s, w}=w_{s}-\xi, e_{s, y}=y_{s}-\xi$. 
Proof similar to Lemma 1 in [8] and Lemma 3.1 in [31].

Theorem 3.2. Let $x_{0}$ be a suitable initial guess to the simple root $\xi$ of $f(x)=0$. Also, suppose the initial values $\gamma_{0}, \beta_{0}$, and $\lambda_{0}$ are chosen properly. Then the $R$-order of the recursive method with memory (3.1) and (3.3) can be obtained from the following system of nonlinear equations:

$\left\{\begin{array}{l}m^{k} m_{1}-\left(1+m_{1}+m_{2}\right)\left(1+m+m^{2}+m^{3}+\ldots+m^{k-1}\right)-m^{k}=0, \\ m^{k} m_{2}-2\left(1+m_{1}+m_{2}\right)\left(1+m+m^{2}+m^{3}+\ldots+m^{k-1}\right)-2 m^{k}=0, \\ m^{k+1}-4\left(1+m_{1}+m_{2}\right)\left(1+m+m^{2}+m^{3}+\ldots+m^{k-1}\right)-4 m^{k}=0,\end{array}\right.$

where $m, m_{1}$ and $m_{2}$ are the order of convergence of the sequences $\left\{x_{k}\right\}_{k=0}^{\infty},\left\{w_{k}\right\}_{k=0}^{\infty}$, and $\left\{y_{k}\right\}_{k=0}^{\infty}$, respectively.

Proof: Let $\left\{x_{k}\right\}_{k=0}^{\infty},\left\{w_{k}\right\}_{k=0}^{\infty}$, and $\left\{y_{k}\right\}_{k=0}^{\infty}$ be convergent successions with orders $m, m_{1}$, and $m_{2}$, respectively. Then:

$$
\left\{\begin{aligned}
e_{k+1} & \sim e_{k}^{m} \sim e_{k-1}^{m^{2}} \sim \ldots \sim e_{0}^{m^{k+1}} \\
e_{k, w} & \sim e_{k}^{m_{1}} \sim e_{k-1}^{m_{1} m} \sim \ldots \sim e_{0}^{m_{1} m^{k}}, \\
e_{k, y} & \sim e_{k}^{m_{2}} \sim e_{k-1}^{m_{2} m} \sim \ldots \sim e_{0}^{m_{2} m^{k}},
\end{aligned}\right.
$$

where $e_{k}=x_{k}-\xi, e_{k, w}=w_{k}-\xi$ and $e_{k, y}=y_{k}-\xi$. Now, by Lemma (3.1) and equation (2.14), we obtain

$$
\begin{aligned}
\left(1+\beta_{k} f^{\prime}(\xi)\right) \sim \prod_{s=0}^{k-1} e_{s} e_{s, w} e_{s, y} & =\left(e_{0} e_{0, w} e_{0, y}\right) \ldots\left(e_{k-1} e_{k-1, w} e_{k-1, y}\right) \\
& =\left(e_{0} e_{0}^{m_{1}} e_{0}^{m_{2}}\right)\left(e_{0}^{m} e_{0}^{m_{1} m} e_{0}^{m_{2} m}\right) \ldots\left(e_{0}^{m^{k-1}} e_{0}^{m^{k-1} m_{1}} e_{0}^{m^{k-1} m_{2}}\right) \\
& =e_{0}^{\left(1+m_{1}+m_{2}\right)+\left(1+m_{1}+m_{2}\right) m+\ldots+\left(1+m_{1}+m_{2}\right) m^{k-1}} \\
& =e_{0}^{\left(1+m_{1}+m_{2}\right)\left(1+m+\ldots+m^{k-1}\right)} .
\end{aligned}
$$

Similarly, we get :

$$
\left(\gamma_{k}+c_{2}\right) \sim e_{0}^{\left(1+m_{1}+m_{2}\right)\left(1+m+\ldots+m^{k-1}\right)},
$$

and

$(3.16)\left(\lambda_{k}+2 f^{\prime}(\xi) c_{2}\left(\gamma_{k}+c_{2}\right)-f^{\prime}(\xi) c_{3}\right) \sim e_{0}^{\left(1+m_{1}+m_{2}\right)\left(1+m+\ldots+m^{k-1}\right)}$. 
By considering the errors of $w_{k}, y_{k}$, and $x_{k+1}$ in equations, (2.8), (2.11) and (2.12), we conclude:

$$
\begin{gathered}
e_{k, w} \sim\left(1+\beta_{k} f^{\prime}(\xi)\right) e_{k} \sim e_{0}^{\left(1+m_{1}+m_{2}\right)\left(1+m+\ldots+m^{k-1}\right)} e_{0}^{m^{k}} \\
e_{k, y} \sim\left(1+\beta_{k} f^{\prime}(\xi)\right)\left(\gamma_{k}+c_{2}\right) e_{k}^{2} \sim e_{0}^{\left(\left(1+m_{1}+m_{2}\right)\left(1+m+\ldots+m^{k-1}\right)\right)^{2}} e_{0}^{2 m^{k}} \\
\quad e_{k+1} \sim \frac{\left.\left(1+\beta_{k} f^{\prime}(\xi)\right)^{2}\left(\gamma_{k}+c_{2}\right)\left(\lambda_{k}+2 f^{\prime}(\xi) c_{2}\left(\gamma_{k}+c_{2}\right)-f^{\prime}(\xi) c_{3}\right)\right)}{f^{\prime}(\xi)} e_{k}^{4} \\
\sim e_{0}^{\left(\left(1+m_{1}+m_{2}\right)\left(1+m+\ldots+m^{k-1}\right)\right)^{4}} e_{0}^{4 m^{k}}
\end{gathered}
$$

Substituting (3.17), (3.18), and (3.19) in (3.13) we get (3.12).

Relationship (3.19) for other weighted functions $g_{2}(t), g_{3}(t), g_{4}(t), g_{5}(t)$, $g_{6}(t)$ and $\left.g_{7}(t)\right)$ will be like $g_{1}(t)$. This completes the proof of the theorem.

Remark 3.3. For $k=1$ the order of convergence of the with memory method can be computed from the following system:

$$
\left\{\begin{array}{l}
m m_{1}-\left(1+m_{1}+m_{2}\right)-m=0, \\
m m_{2}-2\left(1+m_{1}+m_{2}\right)-2 m=0, \\
m^{2}-4\left(1+m_{1}+m_{2}\right)-4 m=0 .
\end{array}\right.
$$

The positive solution of the system (2.18) is given by: $m_{1}=\frac{1}{8}(7+\sqrt{65}), m_{2}=\frac{1}{4}(7+\sqrt{65})$ and $m=\frac{1}{2}(7+\sqrt{65})$.

Therefore, the R-order of the with memory methods (3.1) and (3.3) is at least

$m=\frac{1}{2}(7+\sqrt{65}) \approx 7.53$.

Zafar and colleagues have studied the relation (3.1) for the weight function $g_{2}(t)$ and $k=1$ [31].

Also, for $k=2$, we obtain the order of convergence: $m_{1} \approx 1.98612, m_{2} \approx$ 3.97225 and $m \approx 7.94449$.

For $k=3$, the system of equations(3.12) has the solution: $m_{1} \approx$ $1.99829, m_{2} \approx 3.99657$ and $m \approx 7.99315$. 
Remark 3.4. To obtain the highest efficiency iddex, in what follows, we suggest the best version of the given schemes in (MLTM8). As can be seen, $m$ is near to 8 , which means this with memory method can compete the optimal three-step without memory methods.

$$
\left\{\begin{array}{l}
x_{0}, \gamma_{0}, \beta_{0}, \lambda_{0}, \\
\beta_{k}=-\frac{1}{N_{12}^{\prime}\left(x_{k}\right)}, \gamma_{k}=-\frac{N_{13}^{\prime \prime}\left(w_{k}\right)}{2 N_{13}^{\prime}\left(w_{k}\right)}, \lambda_{k}=\frac{N_{14}^{\prime \prime \prime}\left(y_{k}\right)}{6}, k=4,5,6, \cdots, \\
w_{k}=x_{k}+\beta_{k} f\left(x_{k}\right), y_{k}=x_{k}-\frac{f\left(x_{k}\right)}{f\left[w_{k}, x_{k}\right]+\gamma_{k} f\left(w_{k}\right)}, k=0,1,2, \cdots, \\
x_{k+1}=y_{k}-g_{i}(t) \frac{f\left(x_{k}\right)^{2}}{\left(f\left(x_{k}\right)-f\left(y_{k}\right)\right)^{2}} \frac{f}{f\left[y_{k}, w_{k}\right]+\gamma_{k} f\left(w_{k}\right)+\lambda_{k}\left(y_{k}-x_{k}\right)\left(y_{k}-w_{k}\right)}, i=1,2,5,7
\end{array}\right.
$$

$$
\left\{\begin{array}{l}
x_{0}, \gamma_{0}, \beta_{0}, \lambda_{0}, \\
\beta_{k}=-\frac{1}{N_{12}^{\prime}\left(x_{k}\right)}, \gamma_{k}=-\frac{N_{13}^{\prime \prime}\left(w_{k}\right)}{2 N_{13}^{\prime}\left(w_{k}\right)}, \lambda_{k}=\frac{N_{14}^{\prime \prime \prime}\left(y_{k}\right) N_{14}^{\prime \prime}\left(y_{k}\right)}{12}, k=4,5,6, \cdots, \\
w_{k}=x_{k}+\beta_{k} f\left(x_{k}\right), y_{k}=x_{k}-\frac{f\left(x_{k}\right)}{f\left[w_{k}, x_{k}\right]+\gamma_{k} f\left(w_{k}\right)}, k=0,1,2, \cdots, \\
x_{k+1}=y_{k}-g_{i}(t) \frac{f\left(x_{k}\right)^{2}}{\left(f\left(x_{k}\right)-f\left(y_{k}\right)\right)^{2}} \frac{1}{f\left[y_{k}, w_{k}\right]+\gamma_{k} f\left(w_{k}\right)+\lambda_{k}\left(y_{k}-x_{k}\right)\left(y_{k}-w_{k}\right)}, i=3,4,6,
\end{array}\right.
$$

if $k=4$, we obtain the order of convergence:

$m_{1} \approx 1.99979, m_{2} \approx 3.99957$ and, $m \approx 7.99915$

In this case the efficiency index is $7.99915^{\frac{1}{3}}=1.99993 \approx 2$ which shows that our developed method compets all the existing methods with memory.

\section{Numerical results}

In this section we present results of numerical examples. The existing methods that we are going to compare as follows:

Table 1: Comparison of various iterative methods.

\begin{tabular}{|l|l|}
\hline \hline Ostrowski's method (2) (OM) & Jarratt's method (3) (JM) \\
\hline Maheshwari's method (6)(MM) & Kung-Traub's method (5) (KTM) \\
\hline Cordero et al.'s method (10) (CLTAMM) & Petkovic et al.'s method (8) (PIDM) \\
\hline Kansal et al.'s method (11) (KKBM) & Cordero et al.'s method (9) (CLKTM) \\
\hline Zafar et al.'s method [31] (ZYKZM) & Torkashvand et al.'s method (41,43) (MLTM) \\
\hline
\end{tabular}

The initial value of self-accelerating parameter in all with memory methods is for numerical examples $\left(\beta_{0}=\gamma_{0}=p_{0}=\lambda_{0}=0.1\right)$. The computational software package Mathematica with multi-precision arithmetic has 
been employed. Tables 3,4 and 5 contain absolute values of the errors of approximations in the first three iterations, and, Iter and EI stand for iteration number and efficiency index. These tables contain the entries of the computational order of convergence [19], evaluated by:

$$
r_{c}=\frac{\log \left|f\left(x_{n}\right) / f\left(x_{n-1}\right)\right|}{\log \left|f\left(x_{n-1}\right) / f\left(x_{n-2}\right)\right|} .
$$

A comparison between without memory, with memory and adaptive methods in terms the maximum efficiency index alongside the number of steps per cycle are given in Fig.1.

Figure 1: Comparison of efficiency index of proposed method with other methods.

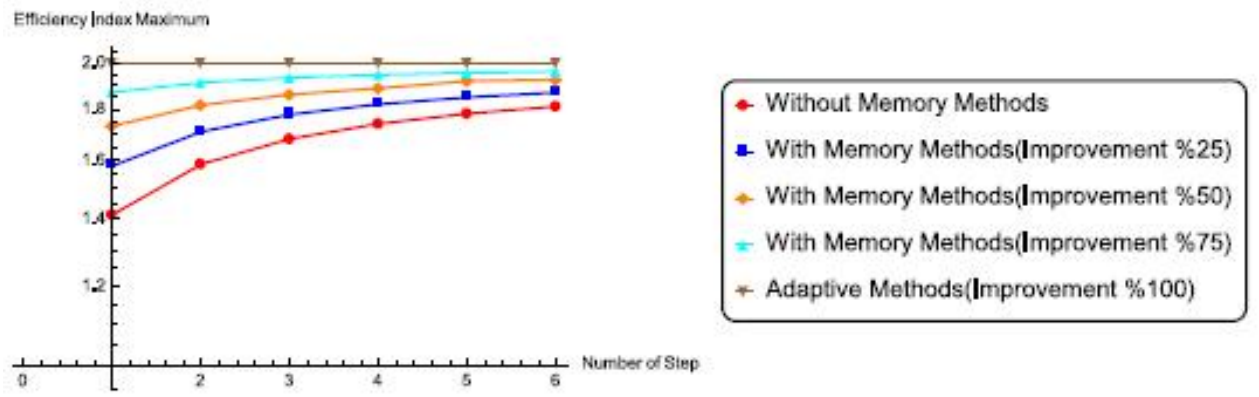

We use the following functions, which are the same as in $[1,2,5,18,30$, $31]$.

Table 2: The test functions

\begin{tabular}{|l|l|l|}
\hline \hline Nonlinear function & Root & Initial guess \\
\hline$f_{1}(x)=x^{5}+x^{4}+4 x^{2}-15[1]$ & $\alpha=1.34743$ & $x_{0}=1.1$ \\
\hline$f_{2}(x)=x^{3}+4 x^{2}-10[2]$ & $\alpha=1.36523$ & $x_{0}=1$ \\
\hline$f_{3}(x)=10 x e^{-x^{2}}-1[5]$ & $\alpha=1.67963$ & $x_{0}=1$ \\
\hline$f_{4}(x)=\sin (5 x) e^{x}-2[18]$ & $\alpha=1.36397$ & $x_{0}=1.5$ \\
\hline$f_{5}(x)=\sin ^{2} x-x^{2}+1[30]$ & $\alpha=1.40449$ & $x_{0}=1$ \\
\hline$f_{6}(x)=e^{x^{2}-3 x} \sin (x)+\log \left(x^{2}+1\right)[31]$ & $\alpha=0.00000$ & $x_{0}=0.35$ \\
\hline
\end{tabular}




\begin{tabular}{|c|c|c|c|c|c|c|}
\hline functions & & $\overline{\mathrm{OM}}$ & JM & KTM & MM & PIDM \\
\hline \multirow[t]{5}{*}{$f_{1}, x_{0}=1.1$} & $\left|x_{n+1}-x_{n}\right|$ & $7.39312 e-35$ & $3.75861 \mathrm{e}-43$ & $5.393380-31$ & $5.77469 \mathrm{e}-72$ & $1.73357 e-751$ \\
\hline & $\left|f\left(x_{n+1}\right)\right|$ & $1.01713 \mathrm{e}-135$ & $4.04445 e-169$ & $5.4005 e-120$ & $1.69766 \mathrm{e}-283$ & $1.79378 e-3339$ \\
\hline & Iter & & 4 & 4 & 5 & 4 \\
\hline & & 4.00000 & 4.00000 & 4.00000 & 4.00000 & 4.44891 \\
\hline & EI & 1.58740 & 1.58740 & 1.58740 & 1.58740 & 1.64469 \\
\hline \multirow[t]{5}{*}{$f_{2}, x_{0}=1$} & $\left|x_{n+1}-x_{n}\right|$ & $2.15826 e-34$ & $3.60235 e-47$ & $3.36806 e-38$ & $3.85254 \mathrm{e}-28$ & $5.35978 \mathrm{e}-1396$ \\
\hline & $\left|f\left(x_{n+1}\right)\right|$ & $5.78606 \mathrm{e}-135$ & $2.45108 \mathrm{e}-186$ & $4.37864 e-64$ & $1.60650 \mathrm{e}-109$ & $0.0000 e-6001$ \\
\hline & Iter & & 4 & 4 & 4 & 4 \\
\hline & $r_{c}$ & 4.00000 & 4.00000 & 4.00000 & 4.00000 & 4.44985 \\
\hline & EI & 1.58740 & 1.58740 & 1.58740 & 1.58740 & 1.64481 \\
\hline \multirow[t]{5}{*}{$f_{3}, x_{0}=1$} & $\left|x_{n+1}-x_{n}\right|$ & $2.15826 e-34$ & $4.40436 e-24$ & $7.32872 \theta-28$ & $3.43019 \mathrm{e}-26$ & $5.68057 e-182$ \\
\hline & $\left|f\left(x_{n+1}\right)\right|$ & $5.78606 \mathrm{e}-135$ & $7.72030 e-94$ & $1.33959 e-108$ & $1.31156 \mathrm{e}-101$ & $1.28915 \mathrm{e}-806$ \\
\hline & & & 4 & 4 & 4 & 4 \\
\hline & $r_{c}$ & 4.0000 & 4.00000 & 4.00000 & 4.00000 & 4.44739 \\
\hline & EI & 1.58740 & 1.58740 & 1.58740 & 1.58740 & 1.64450 \\
\hline \multirow[t]{5}{*}{$f_{4}, x_{0}=1.5$} & $\mid x_{n+1}$ & $7.39312 \mathrm{e}-35$ & $1.24030 e_{-34}$ & $6.17734 e-32$ & $1.50580 \mathrm{e}-26$ & $1.30230 \mathrm{e}-1293$ \\
\hline & $f\left(x_{n-1}\right.$ & $1.01713 e-135$ & $8.78177 e-135$ & $5.11068 e-124$ & $1.91254 \mathrm{e}-102$ & $1.69109 e-5752$ \\
\hline & Iter & & 4 & 4 & 4 & 4 \\
\hline & $r_{c}$ & 4.00000 & 4.00000 & 4.00000 & 4.00000 & 4.45022 \\
\hline & EI & 1.58740 & 1.58740 & 1.58740 & 1.58740 & 1.64485 \\
\hline \multirow[t]{5}{*}{$f_{5}, x_{0}=1$} & $\mid x_{n+}$ & $2.15826 \mathrm{e}-34$ & $3.40910 e-28$ & $6.35886 e-73$ & $1.33500 \mathrm{e}-24$ & $8.49886 e-239$ \\
\hline & $\left|f\left(x_{n+1}\right)\right|$ & $5.78606 \mathrm{e}-135$ & $1.33550 \mathrm{e}-110$ & $3.62654 \mathrm{e}-289$ & $1.46323 \mathrm{e}-95$ & $7.92232 \mathrm{e}-1060$ \\
\hline & Iter & & 4 & 4 & 4 & 4 \\
\hline & $r_{c}$ & 4.00000 & 4.00000 & 4.00000 & 4.00000 & 4.44547 \\
\hline & EI & 1.58740 & 1.58740 & 1.58740 & 1.58740 & 1.64427 \\
\hline \multirow[t]{5}{*}{$f_{6}, x_{0}=0.35$} & $\left|x_{n+1}-x_{n}\right|$ & $2.15826 \mathrm{e}-34$ & $5.53328 e-43$ & $3.45688 \mathrm{e}-33$ & $2.02279 \mathrm{e}-30$ & $3.94711 e-800$ \\
\hline & $\left|f\left(x_{n+1}\right)\right|$ & $5.78606 \mathrm{e}-135$ & $1.71859 \mathrm{e}-169$ & $7.61612 \mathrm{e}-130$ & $3.57162 \mathrm{e}-11$ & $9.16075 \mathrm{e}-3557$ \\
\hline & Iter & & 4 & 4 & 4 & 4 \\
\hline & $r_{c}$ & 4.00000 & 4.00000 & 4.00000 & 4.00000 & 4.44865 \\
\hline & $E I$ & 1.58740 & 1.58740 & 1.58740 & 1.58740 & 1.64466 \\
\hline
\end{tabular}


Table 4: Numerical results.

\begin{tabular}{|c|c|c|c|c|c|c|}
\hline functions & & CLKTM $(b=1)$ & CLTAMM $(A=1)$ & KKBM $(a=1)$ & ZZYKZM, $g_{2}(t)$ & $\bar{T} T M 8, g_{1}(t)$ \\
\hline \multirow[t]{5}{*}{$f_{1}, x_{0}=1.1$} & $\left|x_{n+1}-x_{n}\right|$ & $6.18151 \mathrm{e}-280$ & $2.56524 \mathrm{e}-378$ & $1.16213 e-965$ & $1.04581 \mathrm{e}-188$ & $1.69769 e-267$ \\
\hline & $\left|f\left(x_{n+1}\right)\right|$ & $2.39056 \mathrm{e}-1674$ & $5.79769 e-2643$ & $2.46072 e-6754$ & $1.45636 \mathrm{e}-1416$ & $2.09326 \mathrm{e}-2133$ \\
\hline & & & & & 4 & \\
\hline & $r_{e}$ & 6.00000 & 7.00000 & 7.00000 & 7.52240 & 8.00000 \\
\hline & $E I$ & 1.81712 & 1.91293 & 1.91293 & 1.95938 & 2.00000 \\
\hline \multirow[t]{5}{*}{$f_{2}, x_{0}=1$} & $\left|x_{n+1}-x_{n}\right|$ & $1.49981 e-427$ & $1.94472 \mathrm{e}-908$ & $3.54547 e-778$ & $5.107140-360$ & $2.67370 e-693$ \\
\hline & $\left|f\left(x_{n+1}\right)\right|$ & $3.98170 \mathrm{e}-2562$ & $1.45974 e-7264$ & $1.02498 \mathrm{e}-5444$ & $6.60489 e-2877$ & $3.72689 e-5543$ \\
\hline & & & 4 & & & \\
\hline & $r_{c}$ & 6.00000 & 7.00000 & 7.00000 & 8.00000 & 8.00000 \\
\hline & $E I$ & 1.81712 & 1.91293 & 1.91293 & 2.00000 & 2.00000 \\
\hline \multirow[t]{5}{*}{$f_{3}, x_{0}=1$} & $\left|x_{n+1}-x_{n}\right|$ & $5.18601 e-543$ & $1.69469 e-883$ & $2.19466 \mathrm{e}-964$ & $2.15879 e-1177$ & $5.99959 e-1321$ \\
\hline & $\left|f\left(x_{n+1}\right)\right|$ & $3.17428 \mathrm{e}-3255$ & $2.29452 e-6179$ & $2.37935 e-6744$ & $2.70882 \mathrm{e}-8863$ & $2.11258 e-10563$ \\
\hline & & 4 & 4 & 4 & 4 & \\
\hline & & 6.0000 & 7.00000 & 7.00000 & 7.53226 & 7.99999 \\
\hline & $E I$ & 1.81712 & 1.91293 & 1.91293 & 1.96024 & 2.00000 \\
\hline \multirow[t]{5}{*}{$f_{4}, x_{0}=1.5$} & $\left|x_{n+1}-x_{n}\right|$ & $2.82195 e-195$ & $2.68811 e-200$ & $6.72410 e-333$ & $5.19755 \mathrm{e}-26$ & $7.24241 e-396$ \\
\hline & $\left|f\left(x_{n+1}\right)\right|$ & $9.26117 \mathrm{e}-1174$ & $1.74876 \mathrm{e}-1395$ & $8.22138 \mathrm{e}-2324$ & $2.34517 \mathrm{e}-188$ & $5.74001 \mathrm{e}-3156$ \\
\hline & & 4 & & & 5 & 4 \\
\hline & $r_{c}$ & 6.00000 & 7.00000 & 7.00000 & 7.36349 & 8.00059 \\
\hline & $E I$ & 1.81712 & 1.91293 & 1.91293 & 1.9449 & 2.00005 \\
\hline \multirow[t]{5}{*}{$f_{5}, x_{0}=1$} & $\left|x_{n+1}-x_{n}\right|$ & $4.17283 \mathrm{e}-421$ & $4.64984 \mathrm{e}-1035$ & $9.70878 \mathrm{e}-635$ & $4.37965 \mathrm{e}-92$ & $1.21025 e-736$ \\
\hline & $\left|f\left(x_{n+1}\right)\right|$ & $1.59022 \mathrm{e}-2523$ & $3.22868 e-7242$ & $9.89542 \mathrm{e}-4440$ & $5.46007 e-689$ & $1.62598 \mathrm{e}-5889$ \\
\hline & Iter & & & 4 & 5 & 4 \\
\hline & $r_{0}$ & 6.00000 & 7.00000 & 7.00000 & 7.49554 & 8.00000 \\
\hline & $E I$ & 1.81712 & 1.91293 & 1.91293 & 1.95705 & 2.00000 \\
\hline \multirow[t]{5}{*}{$f_{6}, x_{0}=0.35$} & $\left|x_{n+1}-x_{n}\right|$ & $1.25957 \mathrm{e}-648$ & $5.67578 \mathrm{e}-1381$ & $8.90167 e-1454$ & $7.43034 \mathrm{e}-13642$ & $3.37273 e-2025$ \\
\hline & $\left|f\left(x_{n+1}\right)\right|$ & $1.39931 \mathrm{e}-3886$ & 4. $20564 \mathrm{e}-9662$ & $5.60706 \mathrm{e}-10171$ & $6.06115 \mathrm{e}-102732$ & $4.94141 \mathrm{e}-16194$ \\
\hline & Iter & 4 & 4 & & 5 & \\
\hline & $r_{c}$ & 6.00000 & 7.00000 & 7.00000 & 7.53107 & 8.00000 \\
\hline & $E I$ & 1.81712 & 1.91293 & 1.91293 & 1.96013 & 2.00000 \\
\hline
\end{tabular}


Table 5: Numerical results.

\begin{tabular}{|c|c|c|c|c|c|c|}
\hline functions & & $\bar{T} T M 8, g_{2}(t)$ & $T M 8, g_{3}(t)$ & $T M 8, g_{4}(t)$ & $T M M^{8}, g_{5}(t)$ & $\bar{T} T M 8, g_{6}(t)$ \\
\hline \multirow[t]{5}{*}{$f_{1}, x_{0}=1.1$} & $\left|x_{n+1}-x_{n}\right|$ & $7.29979 \mathrm{e}-200$ & $6.78937 \mathrm{e}-412$ & $1.29484 \mathrm{e}+1$ & $2.77603 \mathrm{e}-208$ & $1.21463 \mathrm{e}-198$ \\
\hline & $\left|f\left(x_{n+1}\right)\right|$ & $2.44592 \mathrm{e}-1592$ & $1.36961 \mathrm{e}-3288$ & $2.62532 \mathrm{e}+5$ & $1.06993 \mathrm{e}-1659$ & $1.43719 \mathrm{e}-1582$ \\
\hline & Iter & 4 & 4 & 4 & & \\
\hline & & 8.00000 & 8.00000 & 8.00000 & 8.00000 & 8.00000 \\
\hline & EI & 2.00000 & 2.00000 & 2.00000 & 2.00000 & 2.00000 \\
\hline \multirow[t]{5}{*}{$f_{2}, x_{0}=1$} & $\left|x_{n+1}-x_{n}\right|$ & $5.10714 e-360$ & $1.14317 e-808$ & $5.0167 \mathrm{e}-897$ & $5.51608 \mathrm{e}-496$ & $4.67598 \mathrm{e}-539$ \\
\hline & $\left|f\left(x_{n+1}\right)\right|$ & $6.60489 \mathrm{e}-2877$ & $4.16240 e-6466$ & $5.67039 e-7173$ & $1.22319 \mathrm{e}-3964$ & $3.26156 \mathrm{e}-4309$ \\
\hline & & 4 & 4 & 4 & 4 & 4 \\
\hline & & 8.00000 & 8.00000 & 8.00000 & 8.00000 & 8.00000 \\
\hline & EI & 2.00000 & 2.00000 & 2.00000 & 2.00000 & 2.00000 \\
\hline \multirow{5}{*}{$f_{3}, x_{0}=1$} & $\left|x_{n+1}-x_{n}\right|$ & $3.00236 \mathrm{e}-543$ & $1.66724 \mathrm{e}-1320$ & $4.49559 \mathrm{e}-1320$ & $2.65708 \mathrm{e}-1316$ & $7.62442 \mathrm{e}-1321$ \\
\hline & $\left|f\left(x_{n+1}\right)\right|$ & $8.30876 \mathrm{e}-10550$ & $7.51329 \mathrm{e}-10560$ & $2.09959 \mathrm{e}-10556$ & $3.12655 e-10526$ & $1.43714 \mathrm{e}-10569$ \\
\hline & & 4 & 4 & 4 & 4 & 4 \\
\hline & & 7.99999 & 7.99999 & 7.99999 & 7.99999 & 7.99999 \\
\hline & EI & 2.00000 & 2.00000 & 2.00000 & 2.00000 & 2.00000 \\
\hline \multirow[t]{5}{*}{$f_{4}, x_{0}=1.5$} & $\left|x_{n+1}-x_{n}\right|$ & $3.47963 \mathrm{e}-394$ & $1.45901 \mathrm{e}-263$ & $1.14126 e-392$ & $6.64310 \mathrm{e}-402$ & $602340 \mathrm{e}-390$ \\
\hline & $f\left(x_{n}\right.$ & $1.62973 \mathrm{e}-3142$ & $2.37315 e-2098$ & $2.18228 \mathrm{e}-3130$ & $2.87634 \mathrm{e}-3204$ & $1.31392 \mathrm{e}-3108$ \\
\hline & & 4 & 4 & 6 & 4 & 4 \\
\hline & & 8.00062 & 7.99984 & 8.00059 & 8.00083 & 8.00053 \\
\hline & EI & 2.00005 & 1.99999 & 2.00005 & 2.00007 & 2.00004 \\
\hline \multirow[t]{5}{*}{$f_{5}, x_{0}=1$} & $\left|x_{n+1}-x_{n}\right|$ & $1.71052 \mathrm{e}-4$ & $8.48791 e-800$ & $4.45891 \mathrm{e}-998$ & $5.17256 \mathrm{e}-520$ & $1.8126 \mathrm{e}-632$ \\
\hline & $\left|f\left(x_{n+1}\right)\right|$ & $2.58905 e-32$ & $9.51757 \mathrm{e}-6395$ & $5.52023 e-7981$ & $1.81031 \mathrm{e}-4156$ & $4.09215 e-5056$ \\
\hline & & & 4 & 4 & & \\
\hline & & 7.22701 & 8.00000 & 8.00000 & 8.00000 & 8.00000 \\
\hline & EI & 1.93339 & 2.00000 & 2.00000 & 2.00000 & 2.00000 \\
\hline \multirow[t]{5}{*}{$f_{6}, x_{0}=0.35$} & $\left|x_{n+1}-x_{n}\right|$ & $3.33535 \mathrm{e}-2025$ & $3.36336 \mathrm{e}-2025$ & $3.35400 \mathrm{e}-20254$ & $3.26154 \mathrm{e}-2025$ & $3.39156 \mathrm{e}-2025$ \\
\hline & $\left|f\left(x_{n+1}\right)\right|$ & $4.51990 \mathrm{e}-16194$ & $4.83262 \mathrm{e}-16194$ & $4.72614 \mathrm{e}-16194$ & $3.77900 \mathrm{e}-16194$ & $5.16648 \mathrm{e}-16194$ \\
\hline & Iter & & & 4 & & \\
\hline & & 8.00000 & 8.00000 & 8.00000 & 8.00000 & 8.00000 \\
\hline & EI & 2.00000 & 2.00000 & 2.00000 & 2.00000 & 2.00000 \\
\hline
\end{tabular}




\section{Conclusion}

In this work, we have developed a new kind of two-step iterative with memory method. To this end, first, we developed an optimal two-step without memory method which uses three functional evaluations and has optimal convergence order four. Then applying three parametes, and accelerating them, without any functional evaluations, the convergence order increased from four to eight, an with memory method. In fact, we increased the efficiency index from 1.58740 to 2 . This improvment means that the developed two-step with memory method can compete any optimal n-step without memory method, since the efficiency index of any optimal without memory method is given by

$$
E I=\lim _{n \rightarrow \infty} 2^{\frac{n}{n+1}}=2,
$$

and the efficiency index of our proposed method is 2 as well.

\section{Acknowledgment}

We would like to deeply thank the editor, Professor Ricardo Soto Montero, as well as the respected referees' for their valuable comments.

\section{References}

[1] N. Choubey, B. Panday, and J. P. Jaiswal, "Several two-point with memory iterative methods for solving nonlinear equations", Afrika matematika, vol. 29, no. 3-4, pp. 435-449, Feb. 2018, doi: $10.1007 / \mathrm{s} 13370-018-0552-\mathrm{x}$

[2] C. Chun, "Some variants of King's fourth-order family of methods for nonlinear equations", Applied mathematics and computation, vol. 190, no. 1, pp. 57-62, Jul. 2007, doi: 10.1016/ j.amc.2007.01.006

[3] C. Chun, "Some fourth-order iterative methods for solving nonlinear equations", Applied mathematics and computation, vol. 195, no. 2, pp. 454-459, Feb. 2008, doi: 10.1016/ j.amc.2007.04.105

[4] C. Chun, M. Y. Lee, B. Neta, and J. Džunić, "On optimal fourth-order iterative methods free from second derivative and their dynamics", Applied mathematics and computation, vol. 218, no. 11, pp. 6427-6438, Feb. 2012, doi: 10.1016/ j.amc.2011.12.013 
[5] A. Cordero, J. L. Hueso, E. Martínez, and J. R. Torregrosa, "New modifications of Potra-Pták's method with optimal fourth and eighth orders of convergence", Journal of computational and applied mathematics, vol. 234, no. 10, pp. 2969-2976, Sep. 2010, doi: 10.1016/ j.cam.2010.04.009

[6] A. Cordero, T. Lotfi, A. Khoshandi, and J. R. Torregrosa, "An efficient Steffensen-like iterative method with memory", Bulletin mathématique de la Société des Sciences Mathématiques de Roumanie Nouvelle Série, vol. 58, no. 1, pp. 49-58, 2015. [On line]. Available: https:// bit.ly/ $3 \mathrm{knrgpO}$

[7] A. Cordero, T. Lotfi, J. R. Torregrosa, P. Assari, and K. Mahdiani, "Some new bi-accelerator two-point methods for solving nonlinear equations", Computational and applied mathematics, vol. 35, no. 1, pp. 251-267, Oct. 2016, doi: 10.1007/s40314-014-0192-1

[8] J. Džunić, "On efficient two-parameter methods for solving nonlinear equations", Numerical algorithms, vol. 63, no. 3, pp. 549-569, Sep. 2013, doi: 10.1007/s11075-012-9641-3

[9] E. Hansen and M. Patrick, "A family of root finding methods", Numerische mathematik, vol. 27 , no. 3, pp. 257-269, Sep. 1976, doi: $10.1007 / \mathrm{bf0} 1396176$

[10] P. Jarratt, "Some fourth order multipoint iterative methods for solving equations", Mathematics of computation, vol. 20, no. 95, pp. 434-434, Sep. 1966, doi: 10.1090/s0025-5718-66-99924-8

[11] M. Kansal, V. Kanwar, and S. Bhatia, "Efficient derivative-free variants of Hansen-Patrick's family with memory for solving nonlinear equations", Numerical algorithms, vol. 73, no. 4, pp. 1017-1036, Mar. 2016, doi: 10.1007/s11075-016-0127-6

[12] R. F. King, "A family of fourth order methods for nonlinear equations", SIAM journal on numerical analysis, vol. 10, no. 5, pp. 876-879, Oct. 1973, doi: $10.1137 / 0710072$

[13] J. Kou, Y. Li, and X. Wang, "A family of fourth-order methods for solving non-linear equations", Applied mathematics and computation, vol. 188, no. 1, pp. 1031-1036, May 2007, doi: 10.1016/ j.amc.2006.10.066

[14] H. T. Kung and J. F. Traub, "Optimal order of one-point and multipoint iteration", Journal of the ACM, vol. 21, no. 4, pp. 643-651, Oct. 1974, doi: $10.1145 / 321850.321860$

[15] T. Lotfi and P. Assari, "New three- and four-parametric iterative with memory methods with efficiency index near 2", Applied mathematics and computation, vol. 270, pp. 1004-1010, Nov. 2015, doi: 10.1016/ j.amc.2015.08.017

[16] A. K. Maheshwari, "A fourth order iterative method for solving nonlinear equations", Applied mathematics and computation, vol. 211, no. 2, pp. 383-391, May 2009, doi: 10.1016/ j.amc.2009.01.047 
[17] A. M. Ostrowski, Solution of equations and systems of equations. Englew ood Ciffs, NJ: Prentice-Hall, 1964

[18] M. S. Petković, S. Ilić, and J. Džunić, "Derivative free two-point methods with and without memory for solving nonlinear equations", Applied mathematics and computation, vol. 217, no. 5, pp. 1887-1895, Nov. 2010, doi: 10.1016/ j.amc.2010.06.043

[19] M. Petkovic, B. Neta, L. Petkovic and J. Dzunic, Multipoint methods for solving nonlinear equations. Amsterdam: Elsevier, 2013.

[20] M. Sharifi, D. Babajee, and F. Soleymani, "Finding the solution of nonlinear equations by a class of optimal methods", Computers \& mathematics with applications, vol. 63, no. 4, pp. 764-774, Feb. 2012, doi: 10.1016/ j.camwa.2011.11.040

[21] J. R. Sharma, R. K. Guha, and P. Gupta, "Some efficient derivative free methods with memory for solving nonlinear equations", Applied mathematics and computation, vol. 219, no. 2, pp.699-707, Oct. 2012, doi: $10.1016 /$ j.amc.2012.06.062

[22] F. Soleymani, R. Sharma, X Li, and E. Tohidi, "An optimized derivativefree form of the Potra-Pták method", Mathematical and computer modelling, vol. 56, no. 5-6, pp. 97-104, Sep. 2012, doi: $10.1016 /$ j.mcm.2011.12.005

[23] F. Soleymani, S. Khattri, and S. K. Vanani, "Two new classes of optimal Jarratt-type fourth-order methods", Applied mathematics letters, vol. 25, no. 5, pp. 847-853, May 2012, doi: 10.1016/ j.aml.2011.10.030

[24] F. Soleymani and V. Hosseinabadi, "Robust cubically and quartically iterative techniques free from derivative", Proyecciones (Antofagasta), vol. 30, no. 2, pp. 149-161, Dec. 2011, doi: $10.4067 / \mathrm{s} 0716-09172011000200002$

[25] V. Torkashvand, T. Lotfi, and M. A. Fariborzi Araghi, "Efficient iterative methods with memory for solving nonlinear equations" in 49-Annual Iranian Mathematics Conference, 2018.

[26] V. Torkashvand, T. Lotfi, and M. A. F. Araghi, "A new family of adaptive methods with memory for solving nonlinear equations", Mathematical sciences, vol. 13, no. 1, pp. 1-20, Dec. 2018, doi: $10.1007 / \mathrm{s} 40096-018-0272-2$

[27] J. F. Traub, Iterative methods for the solution of equations. Englewood Cliffs, NJ: Prentice-Hall, 1964.

[28] X Wang, "An Ostrowski-type method with memory using a novel self-accelerating parameter", Journal of computational and applied mathematics, vol. 330, pp. 710-720, Mar. 2018, doi: 10.1016/ j.cam.2017.04.021 
[29] X. Wang and T. Zhang, "A new family of Newton-type iterative methods with and without memory for solving nonlinear equations", Calcolo, vol. 51, no. 1, pp.1-15, Sep. 2014, doi: 10.1007/s10092-012-0072-2

[30] Z. Xiaojian, "Modified Chebyshev-Halley methods free from second derivative", Applied mathematics and computation, vol. 203, no. 2, pp. 824-827, Sep. 2008, doi: 10.1016/ j.amc.2008.05.092

[31] F. Zafar, N. Yasmin, M. A. Kutbi, and M. Zeshan, "Construction of Tri-parametric derivative free fourth order with and without memory iterative method", Journal of nonlinear sciences and applications, vol. 09, no. 04, pp. 1410-1423, Apr. 2016, doi: 10.22436/ jnsa.009.04.01 